\title{
Usefulness of the Whole Blood Passage Time as a Predictor of Primary Cardiovascular Events in Patients With Traditional Cardiovascular Risk Factors
}

\author{
Takashi Hitsumoto
}

\begin{abstract}
Background: Recent clinical studies have reported that impaired hemorheology is a significant cardiovascular risk factor, but there has been no prospective study of its relationship with cardiovascular events. The aim of this prospective study was to assess the efficacy of whole blood passage time (WBPT), measured by a microchannel array flow analyzer (MC-FAN), as a predictor of primary cardiovascular events in patients with traditional cardiovascular risk factors.
\end{abstract}

Methods: The study enrolled 1,134 outpatients with traditional cardiovascular risk factors but no history of cardiovascular events (438 men and 696 women; mean \pm standard deviation age, $67 \pm 11$ years). Based on the value of WBPT, the patients were assigned to one of three groups: L (low, WBPT $<50 \mathrm{~s} ; \mathrm{n}=499$ ), $\mathrm{M}$ (medium, WBPT $50-70 \mathrm{~s} ; \mathrm{n}=295$ ), or H (high, WBPT $>70 \mathrm{~s} ; \mathrm{n}=340$ ). The utility of the WBPT as a predictor of primary cardiovascular events was evaluated.

Results: During the follow-up period (median 81.9 months), major adverse cardiovascular events (MACE) occurred in 95 cases (L, 21 cases $(4.2 \%)$; M, 24 cases $(8.1 \%) ; \mathrm{H}, 50$ cases $(14.7 \%)$; $\mathrm{P}<0.001$, log-rank test). In multivariate Cox regression analyses, the risk for MACE was significantly higher in group $\mathrm{H}$ than in group L (hazard ratio, 2.32; 95\% confidence interval, $1.31-3.20 ; \mathrm{P}<0.01)$. A WBPT cut-off of $72.4 \mathrm{~s}$ yielded the largest area under the curve of 0.705 ( $95 \%$ confidence interval: $0.678-0.732$ ), with a sensitivity of $51.7 \%$ and specificity of $85.4 \%$ for discriminating between those who did and did not experience MACE during the follow-up period.

Conclusion: This study showed that WBPT evaluated by a MC-FAN was a predictor of primary cardiovascular events in patients with traditional cardiovascular risk factors.

Keywords: Hemorheology; Whole blood passage time; Microchannel array flow analyzer; Cardiovascular risk factor; Primary cardiovascular events; Cardio-ankle vascular index

Manuscript submitted July 26, 2018, accepted August 3, 2018

Hitsumoto Medical Clinic, 2-7-7, Takezakicyou, Shimonoseki City, Yamaguchi, 750-0025, Japan. Email: thitsu@jcom.home.ne.jp

doi: https://doi.org/10.14740/cr763w

\section{Introduction}

In the management of outpatients, reducing the risk of cardiovascular event is based mainly on addressing traditional cardiovascular risk factors such as hypertension, diabetes mellitus, dyslipidemia, obesity, and smoking habits. However, although these risk factors are important, they do not explain every cardiovascular event [1-3]. It is therefore important to explore novel biomarkers of cardiovascular diseases.

The impairment of hemorheology is considered to be a further important factor in the incidence of cardiovascular events, in addition to atherosclerosis $[4,5]$. In recent years, a commercial device to evaluate hemorheology using microscopic images, the microchannel array flow analyzer (MC-FAN), has been introduced to the clinical setting [6]. The MC-FAN is simple and is superior to other methods of hemorheological evaluation in terms of the accuracy of channel dimensions and high reproducibility. MC-FAN can be used to measure the whole blood passage time (WBPT). Cross-sectional studies have reported significant relationships between an increase in WBPT and cardiovascular risk factors or cardiovascular disease [7-10]. However, there have been no prospective studies of the clinical usefulness of WBPT as a predictor of cardiovascular events. The aim of this prospective study was therefore to assess the efficacy of WBPT evaluated by the MC-FAN as a predictor of primary cardiovascular events in patients with traditional cardiovascular risk factors.

\section{Materials and Methods}

\section{Participants}

Between January 2008 and December 2009, 1,134 outpatients (696 women (61.4\%) and 438 men (38.6\%)) with traditional cardiovascular risk factors but no history of cardiovascular events were prospectively enrolled at the Hitsumoto Medical Clinic, Yamaguchi, Japan. The mean ( \pm standard deviation) age was $67 \pm 11$ years. WBPT was measured as described below and the participants were assigned accordingly to one of three groups: L (low, WBPT $<50 \mathrm{~s} ; \mathrm{n}=499$ ), M (medium, $\mathrm{WBPT}=50-70 \mathrm{~s} ; \mathrm{n}=295)$, or H (high, WBPT $>70 \mathrm{~s} ; \mathrm{n}=$ 340). The study protocol was approved by Local Ethics Com- 
mittee of Hitsumoto Medical Clinic and informed consent was obtained from all the participants.

\section{Evaluation of hemorheology using an MC-FAN}

The participant's hemorheology was evaluated by measuring WBPT using an MC-FAN HR300 rheometer (MC Healthcare Inc., Tokyo), as previously described $[6,8]$. Briefly, the microchannel passage time for $100 \mu \mathrm{L}$ of physiologic saline was first measured as a control. Then, the same measurement was determined for blood obtained from the participant with $100 \mu \mathrm{L}$ of the heparinization sample. The WBPT was corrected for the passage time of the physiologic saline, SPT, as WBPT $\times 12 /$ SPT. The flow of blood cells through individual microchannels was observed and recorded using an inverted metallographic microscope, video camera, and video recorder. The width, length, and depth of the microchannel formation were 7, 30, and $4.5 \mu \mathrm{m}$, respectively. Examination was performed within $60 \mathrm{~min}$ of blood sampling. The inter- and intra-assay coefficients of variation for WBPT were $8 \%$ and $5 \%$, respectively.

\section{Evaluation of clinical parameters}

The participant's body mass index was calculated as the weight in kilograms divided by the square of the height in meters; obesity was defined by the Japanese criteria of body mass index $\geq 25 \mathrm{~kg} / \mathrm{m}^{2}$. Current smoking was defined as smoking at least one cigarette per day during the previous 28 days. Hypertension was defined as systolic blood pressure $\geq 140$ $\mathrm{mm} \mathrm{Hg}$, diastolic blood pressure $\geq 90 \mathrm{~mm} \mathrm{Hg}$, or the use of anti-hypertensive medication. Dyslipidemia was defined as a low-density lipoprotein cholesterol level $\geq 140 \mathrm{mg} / \mathrm{dL}$, a highdensity lipoprotein cholesterol level $\leq 40 \mathrm{mg} / \mathrm{dL}$, a triglyceride level $\geq 150 \mathrm{mg} / \mathrm{dL}$, or the use of anti-dyslipidemic medication. Diabetes mellitus was defined as a fasting blood glucose level $\geq 126 \mathrm{mg} / \mathrm{dL}$ or the use of anti-diabetic medication. The following blood parameters were measured: blood cell counts, plasma glucose, serum lipid concentrations, and serum highsensitivity C-reactive protein (hs-CRP) concentration. Blood samples were collected from the antecubital vein in the morning after $12 \mathrm{~h}$ of fasting. Total cholesterol and triglyceride concentrations were measured using standard enzymatic methods. High- and low-density lipoprotein cholesterol concentrations were measured using selective inhibition and Friedewald's formula, respectively [11]. Participants with a serum triglyceride concentration $\geq 400 \mathrm{mg} / \mathrm{dL}$ were excluded from the analysis, considering the accuracy of this method. Glucose concentrations were measured by the glucose oxidase method. The hsCRP concentration was measured using high-sensitivity latexenhanced immunonephelometry. As a physiological marker of arterial function, the cardio-ankle vascular index (CAVI) was measured using a VaSera CAVI system (Fukuda Denshi), as described previously [12]. Briefly, brachial and ankle pulse waves were determined using inflatable cuffs, with the pressure maintained between 30 and $50 \mathrm{~mm} \mathrm{Hg}$ to ensure that the cuff pressure exerted minimal effects on systemic hemo- dynamics. Of note, systemic blood and pulse pressures were simultaneously determined with the participants in the supine position. CAVI was measured after a 10 -min rest in a quiet room. The value used for CAVI was the mean of the values for the left and right sides.

\section{Follow-up}

For this study, the follow-up period terminated in January 2018. The endpoint for this study was a major adverse cardiovascular event (MACE), a composite of cardiovascular death, non-fetal myocardial infarction, and non-fetal ischemic stroke. The median follow-up period to determine the incidence of MACE was 81.9 months (range, 4 - 120 months).

\section{Statistical analysis}

Data were analyzed using Stat View-J 5.0 (HULINKS, Tokyo, Japan) and MedCalc for Windows version 14.8.1 (MedCalc Software, Ostend, Belgium). Data are presented as mean \pm standard deviation. One-way analysis of variance (ANOVA) and the Kruskal-Wallis test were used for comparisons of the three groups. Post-hoc testing was performed using Fisher's protected least significant differences or the Mann-Whitney U-test with the Bonferroni correction. Event-free survival rate curves were plotted using Kaplan-Meier analysis and the differences between the curves were evaluated using the longrank test. Multivariate analysis was performed using multivariate Cox regression analysis. Receiver operating characteristic (ROC) curves were constructed and the Youden Index was used to determine the optimal cut-off for WBPT for predicting the participants who experienced a MACE. $\mathrm{P}<0.05$ was considered statistically significant.

\section{Results}

Table 1 presents the characteristics of the participants at registration. The mean WBPT for groups L, M, and H were 40.3, 59.6 , and $79.4 \mathrm{~s}$, respectively. The following factors were significantly higher in $\mathrm{H}$ than in $\mathrm{M}$ or $\mathrm{L}$ : the proportions of men, current smokers, and participants with diabetes mellitus, and the mean hematocrit, fasting blood glucose concentration, hs-CRP concentration, and CAVI values. Figure 1 shows the Kaplan-Meier curve for the incidence of MACE. The median follow-up period was 81.9 months. During follow-up, 95 participants experienced at least one MACE (L, 21 participants (4.2\%); M, 24 participants (8.1\%); H, 50 participants $(14.7 \%))$. The Kaplan-Meier curve confirmed that group $\mathrm{H}$ had a higher incidence of MACE compared to groups M and L (log-rank test, $\mathrm{P}<0.001)$. Table 2 presents the clinical parameters at registration of all the participants, comparing those who experienced MACEs with those who did not. Male sex, age, current smoking status, systolic blood pressure, the presence of diabetes mellitus, fasting blood glucose concentration, hs-CRP concentration, and CAVI were considerably higher in partici- 
Table 1. Characteristics of Patients

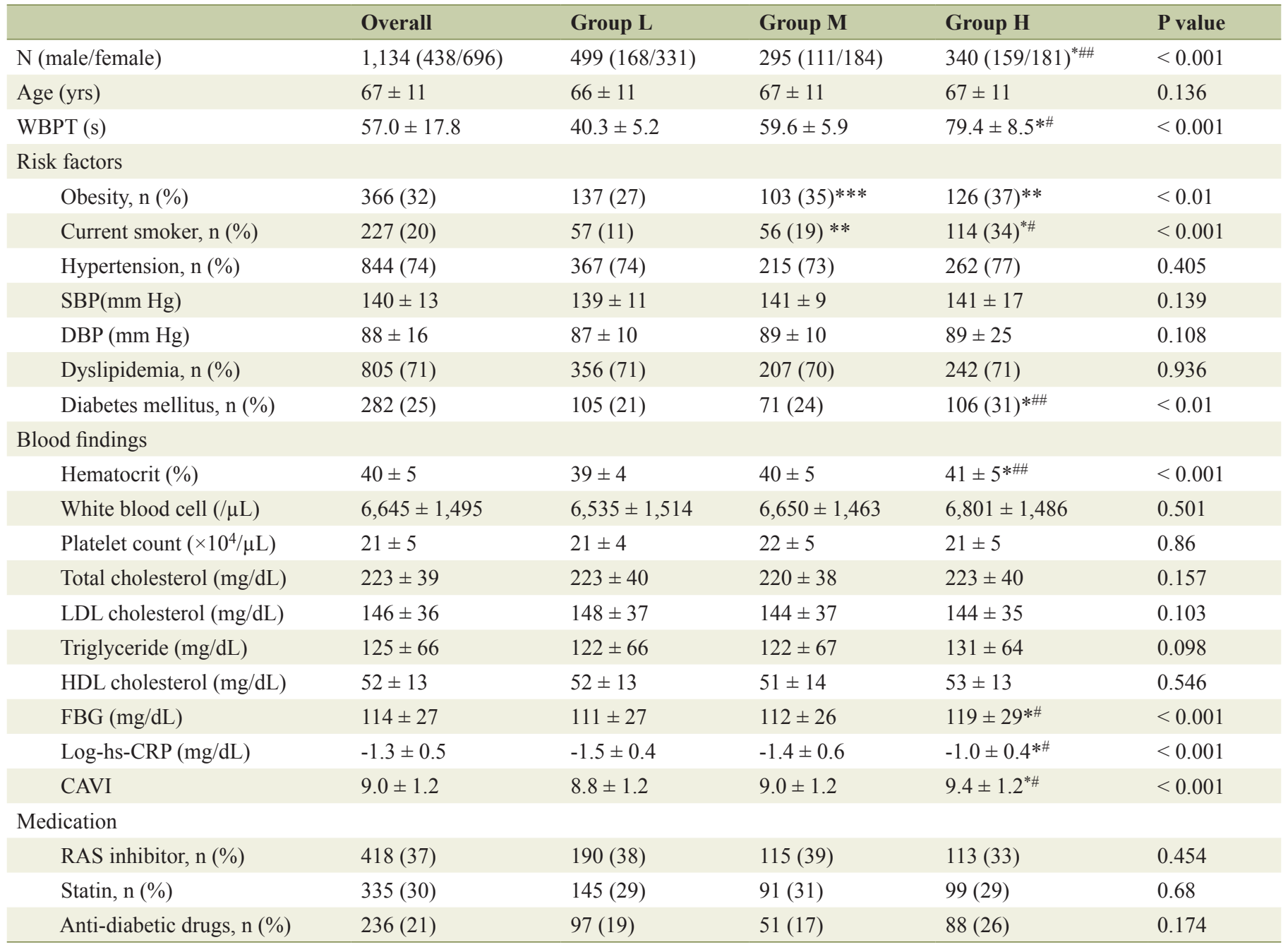

Continuous values are mean \pm SD. WBPT: whole blood passage time; SBP: systolic blood pressure; DBP: diastolic blood pressure; LDL: low-density lipoprotein; HDL: high-density lipoprotein; FBG: fasting blood glucose; hs-CRP: high sensitivity C reactive protein; CAVI: cardio-ankle vascular index; RAS: renin-angiotensin system. ${ }^{*} P<0.001$ vs. group $L$, ${ }^{* *} P<0.01$ vs. group $L,{ }^{* * *} P<0.05$ vs. group $L$, ${ }^{\#} P<0.001$ vs. group $M$, ${ }^{\# \#} P<0.05$ vs. group $M$.

pants who experienced MACE than in those who did not, and levels of renin-angiotensin system (RAS) inhibitor and statin use were considerably lower. Table 3 presents the results of the multivariate Cox regression analysis for the MACE. The 10 variables were selected as being significant factors for MACE in the univariate analysis or check of multicollinearity among variables. Of these, seven variables (CAVI, being in group $\mathrm{H}$, male sex, age, the presence of diabetes mellitus, hs-CRP concentration, and statin use) exhibited a considerable hazard ratio (HR) for MACE. Figure 2 shows the ROC curve analysis for the incidence of MACE based on WBPT. A cut-off value for WBPT of $72.4 \mathrm{~s}$ yielded the largest area under the curve of 0.705 (95\% confidence interval (CI): $0.678-0.732)$, with a sensitivity of $51.7 \%$ and specificity of $85.4 \%$ for discriminating between the participants who did and did not experience MACE during the follow-up period. To assess the clinical efficacy of a combination of the biomarkers for hemorheol- ogy and arterial function, the participants were divided into four groups according to cut-off levels of WBPT $=72.4 \mathrm{~s}$ and $\mathrm{CAVI}=9$, and a multivariate Cox regression analysis was performed (Fig. 3). Having values above the cut-off for one of these factors (WBPT $>73.4 \mathrm{~s}$ or CAVI $>9$ ) was associated with significantly higher HRs for MACE (HR: 3.18, 95\% CI: 1.29 - 7.44, P $<0.01$; HR: 3.36, 95\% CI: 1.31 - 7.60, $\mathrm{P}<0.01$, respectively) than having values below these cut-offs. The HR was higher still when both factors were above the cut-off levels (HR, 10.62; 95\% CI, $5.38-21.31$; $<0.001)$ compared with both factors being below the cut-offs.

\section{Discussion}

Previous studies have reported relationships between the incidence of cardiovascular disease and traditional risk factors 


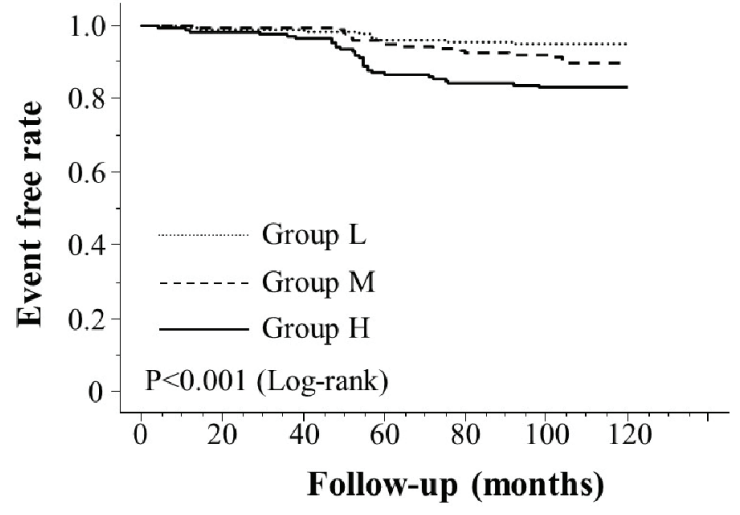

Figure 1. Kaplan-Meier curve for the incidence of major adverse cardiovascular events. The Kaplan-Meier curve confirmed that group $\mathrm{H}$ had a higher incidence of major adverse cardiovascular events compared to groups $M$ and $L$ (log-rank test, $P<0.001$ ).

such as male sex, aging, and diabetes mellitus [13-15]. Consistent with these previous findings, the results of the present study found these factors to be independent predictors for MACE. In addition, this study found that high WBPT was a further independent predictor for MACE. WBPT showed sig-
Table 3. Multivariate Cox Regression Analysis for Major Adverse Cardiovascular Event

\begin{tabular}{llll} 
HR & & $\mathbf{9 5 \%}$ CI & P value \\
\hline CAVI ( $>$ 9) & 2.52 & $1.35-3.53$ & $<0.01$ \\
Group H (vs. group L) & 2.32 & $1.31-3.20$ & $<0.01$ \\
Sex (male) & 2.05 & $1.30-3.13$ & $<0.01$ \\
Age ( $\geq 65$ yrs) & 1.87 & $1.15-3.07$ & $<0.01$ \\
Diabetes mellitus & 1.69 & $1.11-2.57$ & $<0.05$ \\
hs-CRP $(\geq 0.1 \mathrm{mg} / \mathrm{dL})$ & 1.45 & $1.04-2.22$ & $<0.05$ \\
Statin & 0.67 & $0.33-0.92$ & $<0.05$ \\
Current smoker & 1.38 & $0.98-2.01$ & 0.065 \\
RAS inhibitor & 0.66 & $0.42-1.04$ & 0.073 \\
SBP $(\geq 140 \mathrm{~mm} \mathrm{Hg)}$ & 1.23 & $0.80-1.91$ & 0.339 \\
\hline
\end{tabular}

HR: hazard ratio; $\mathrm{Cl}$ : confidence interval; CAVI: cardio-ankle vascular index; hs-CRP: high sensitivity $C$ reactive protein; RAS: renin-angiotensin system; SBP: systolic blood pressure.

nificant associations with smoking habits, hs-CRP concentration, and CAVI. The study also indicated the clinical efficacy of using a combination of the WBPT and CAVI as a predictor

Table 2. Clinical Parameters at Registration of Patients With and Without Major Adverse Cardiovascular Event

\begin{tabular}{|c|c|c|c|}
\hline & MACE (-) & MACE (+) & P value \\
\hline $\mathrm{N}$ (male/female) & $1,039(385 / 654)$ & $95(53 / 42)$ & $<0.001$ \\
\hline Age (yrs) & $66 \pm 11$ & $70 \pm 9$ & $<0.01$ \\
\hline Current smoker, n (\%) & $197(19)$ & $30(32)$ & $<0.01$ \\
\hline Hypertension, $\mathrm{n}(\%)$ & $772(74)$ & $72(76)$ & 0.751 \\
\hline Dyslipidemia, n (\%) & $738(71)$ & $67(71)$ & 0.918 \\
\hline Diabetes mellitus, n (\%) & $242(23)$ & $40(42)$ & $<0.001$ \\
\hline Hematocrit (\%) & $40 \pm 5$ & $41 \pm 5$ & 0.640 \\
\hline White blood cell $(/ \mu \mathrm{L})$ & $6,624 \pm 1,492$ & $6,884 \pm 1,484$ & 0.102 \\
\hline Triglyceride (mg/dL) & $125 \pm 66$ & $125 \pm 64$ & 0.509 \\
\hline HDL cholesterol (mg/dL) & $52 \pm 13$ & $50 \pm 13$ & 0.146 \\
\hline $\mathrm{FBG}(\mathrm{mg} / \mathrm{dL})$ & $113 \pm 27$ & $121 \pm 27$ & $<0.05$ \\
\hline Log-hs-CRP (mg/dL) & $-1.3 \pm 0.6$ & $-1.1 \pm 0.5$ & $<0.001$ \\
\hline CAVI & $9.0 \pm 1.2$ & $9.9 \pm 1.1$ & $<0.001$ \\
\hline RAS inhibitor, $\mathrm{n}(\%)$ & $393(38)$ & $25(26)$ & $<0.05$ \\
\hline Statin, n (\%) & $318(31)$ & $17(18)$ & $<0.01$ \\
\hline Anti-diabetic drug, n (\%) & $210(20)$ & $26(27)$ & 0.100 \\
\hline
\end{tabular}

Continuous values are mean \pm SD. SBP: systolic blood pressure; DBP: diastolic blood pressure; LDL: low-density lipoprotein; HDL: high-density lipoprotein; FBG: fasting blood glucose; hs-CRP: high sensitivity C reactive protein; CAVI: cardio-ankle vascular index; RAS: renin-angiotensin system. 


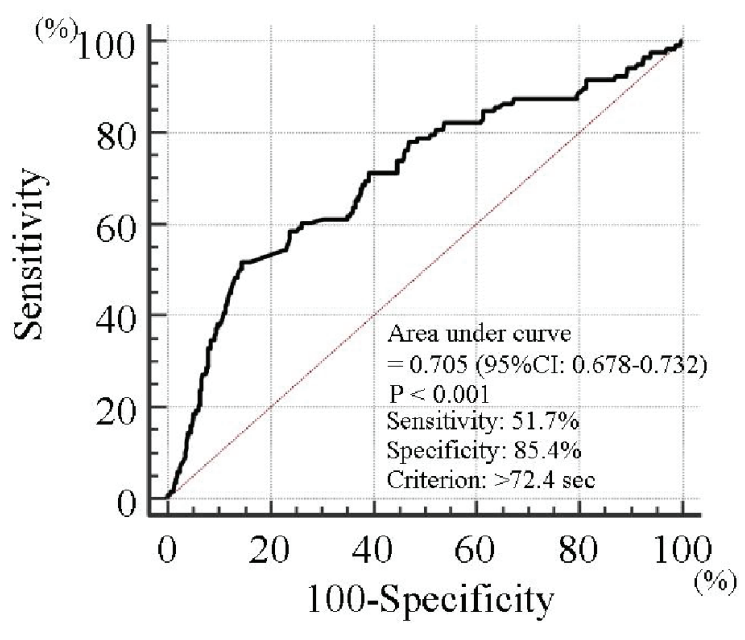

Figure 2. Prediction of major adverse cardiovascular events at followup period using WBPT. A WBPT cut-off of $72.4 \mathrm{~s}$ yielded the largest area under the curve of 0.705 (95\% confidence interval: $0.678-0.732$ ), with a sensitivity of $51.7 \%$ and specificity of $85.4 \%$ for discriminating between those who did and did not experience major adverse cardiovascular events during the follow-up period. WBPT: whole blood passage time.

\section{for MACE.}

Several cross-sectional studies have assessed WBPT in healthy populations or in patients with cardiovascular risk factors, with the results suggesting that the cut-off value for cardiovascular risk is $50-70 \mathrm{~s}$ [10, 16-18]. Based on these previous results, the present study prospectively divided the participants into three groups according to two simple cut-off levels of 50 $\mathrm{s}$ and $70 \mathrm{~s}$. The results showed that participants with WBPT $>$ $70 \mathrm{~s}$ were a population at high risk of primary cardiovascular events. In the ROC analysis, a WBPT cut-off of $72.4 \mathrm{~s}$ yielded the largest area under the curve of 0.705 for discriminating between those who did and did not experience MACE during the follow-up period. Thus, for primary cardiovascular disease prevention, we perform examinations or intervention therapy for any patients with WBPT exceeding approximately $70 \mathrm{~s}$.

There are several possible mechanisms to explain why smoking affects hemorheology, such as platelet activation, an increase in leukocyte adhesion ability, and elevation of plasma viscosity $[19,20]$. There have been several clinical studies of the relationship between smoking habits and WBPT $[8,16$, $18,21]$. Shimada et al. reported a positive correlation between WBPT and the daily consumption of tobacco or the Brinkman index, and three months of smoking cessation significantly reduced WBPT [21]. In contrast, in this study the prevalence of smoking increased with an increase in WBPT. Even though a smoking habit was not found to be an independent predictor for MACE in this study, the results of this and previous studies have indicated that smoking cessation is strongly recommended to improve hemorheology.

Hs-CRP is used as a marker of inflammation, and several epidemiological studies have indicated that a high hs-CRP lev$\mathrm{el}$ is a predictor of cardiovascular disease $[22,23]$. The results of the present study also found that high hs-CRP $(\geq 0.1 \mathrm{mg} /$ dL) was an independent predictor for MACE in patients with

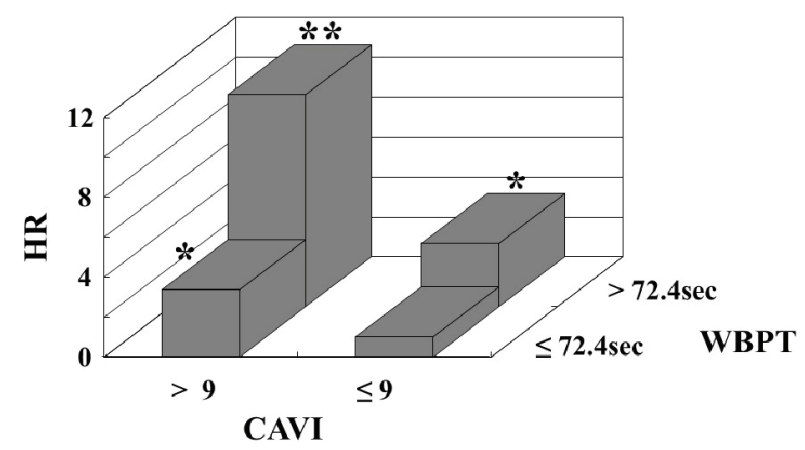

Figure 3. Multivariate Cox regression analysis for major adverse cardiovascular events using a combination of the WBPT and CAVI. The participants were divided into four groups according to cut-off levels of $\mathrm{WBPT}=72.4 \mathrm{~s}$ and $\mathrm{CAVI}=9$, and a multivariate Cox regression analysis was performed. Having values above the cut-off for one of these factors (WBPT > $73.4 \mathrm{~s}$ or CAVI > 9) was associated with significantly higher HRs for major adverse cardiovascular events (HR: $3.18,95 \%$ Cl: 1.29 - 7.44, P< 0.01; HR: 3.36, 95\% Cl: 1.31 - 7.60, P<0.01, respectively) than having values below these cut-offs. The HR was higher still when both factors were above the cut-off levels (HR, 10.62; 95\% $\mathrm{Cl}, 5.38-21.31 ; \mathrm{P}<0.001)$ compared with both factors being below the cut-offs. Adjustment factors are sex, age, diabetes mellitus, hs-CRP, and statin use. ${ }^{*} \mathrm{P}<0.01$ vs. patients with WBPT as $\leq 72.4 \mathrm{~s}$ and CAVI as $\leq 9$; ${ }^{* *} \mathrm{P}<0.001$ vs. patients with WBPT as $\leq 72.4$ s and $\mathrm{CAVI}$ as $\leq 9$. WBPT: whole blood passage time; CAVI: cardio-ankle vascular index; HR: hazard ratio, $\mathrm{Cl}$ : confidence interval; hs-CRP: high sensitivity $\mathrm{C}$ reactive protein.

traditional cardiovascular risk factors. One explanation for hsCRP level being a cardiovascular risk factor is thought to be chronic inflammation in the arterial walls, which contributes to the development of atherosclerosis, including plaque instability [24-26]. Several mechanisms by which inflammation causes impairment of blood rheology have been proposed, including platelet aggregation and the elevation of plasma viscosity [27, 28]. In this study, the hs-CRP levels were significantly higher in group $\mathrm{H}$ than in groups $\mathrm{M}$ and L. RAS inhibitors and statins have been reported to reduce inflammation in vivo [29-31], with a reported reduction in the incidence of primary cardiovascular events $[32,33]$. However, the use of medications such as RAS inhibitors or statins was approximately $30 \%$ degree in group $\mathrm{H}$. Positively using such drugs with anti-inflammatory effects for patients with high hs-CRP concentration, especially those with high WBPT, may help reduce the incidence of cardiovascular.

CAVI provides a novel marker of systemic arterial stiffness that is independently associated with blood pressure [12]. A number of studies have reported the clinical usefulness of CAVI as a cardiovascular risk factor [34-36]. In the present study, CAVI $>9$ was one of the strongest predictors of MACE of all the explanatory variables. In addition, CAVI levels were higher in the participants with higher WBPT values. There have been several reports of a significant relationship between WBPT and physiological markers of arterial stiffness [37, 38]. In addition, a study has reported a significant relationship between WBPT and endothelial dysfunction [39], and Endo et al reported that CAVI reflected endothelial dysfunction as es- 
timated by brachial artery flow-mediated vasodilatation [40]. Thus, the results of this and previous studies suggest that impaired hemorheology affected arterial function such as arterial stiffness or endothelial dysfunction, thereby increasing the incidence of cardiovascular disease.

To evaluate the clinical efficacy of using a combination of hemorheology and arterial function biomarkers, the participants of this study were divided into four groups based on the cut-off values of WBPT $=72.4 \mathrm{~s}$ and $\mathrm{CAVI}=9$ and a multivariate analysis was performed. The HR for experiencing a MACE with both high WBPT and high CAVI compared with both low WBPT and low CAVI was approximately 3 points higher than the HR for either high WBPT or high CAVI but not both. Thus, the participants with high WBPT and CAVI were considered to be a high-risk population for primary cardiovascular events. Previous studies have shown that lifestyle or medical interventions affected both hemorheology and arterial function [8, 21, 41-46], and the methods for measuring WBPT and CAVI are simple and take little time in clinical practice. We therefore check these two markers in our patients and administer intervention therapy such as lifestyle modification or medication for those with high WBPT and CAVI. In this way, we expect to efficiently reduce primary cardiovascular events in patients with traditional cardiovascular risk factors.

\section{Limitations}

This study had several limitations. First, it was conducted at a single center and its findings cannot be generalized to all medical centers. Second, WBPT was measured only once, at registration. A further investigation of the association between serial changes in WBPT and primary cardiovascular events is needed. Finally, further studies concerning patients with high WBPT and traditional cardiovascular risk factors are warranted to determine whether aggressive intervention therapy, such as lifestyle modification or medication, reduces the incidence of primary cardiovascular events.

\section{Conclusions}

This study demonstrated that WBPT evaluated by MC-FAN as a marker of hemorheology was a predictor of primary cardiovascular events in patients with traditional cardiovascular risk factors. The predictive value for the incidence of cardiovascular events was increased by using a combination of WBPT and CAVI as a marker of arterial function.

\section{Conflict of Interest}

The author has reported no conflict of interest.

\section{Grant Support}

None.

\section{Disclosures}

The author has no competing interest.

\section{References}

1. Noda H, Iso H, Irie F, Sairenchi T, Ohtaka E, Ohta H. Gender difference of association between LDL cholesterol concentrations and mortality from coronary heart disease amongst Japanese: the Ibaraki Prefectural Health Study. J Intern Med. 2010;267(6):576-587.

2. Takashima N, Ohkubo T, Miura K, Okamura T, Murakami Y, Fujiyoshi A, Nagasawa SY, et al. Long-term risk of BP values above normal for cardiovascular mortality: a 24-year observation of Japanese aged 30 to 92 years. J Hypertens. 2012;30(12):2299-2306.

3. Stratton IM, Adler AI, Neil HA, Matthews DR, Manley SE, Cull CA, Hadden D, et al. Association of glycaemia with macrovascular and microvascular complications of type 2 diabetes (UKPDS 35): prospective observational study. BMJ. 2000;321(7258):405-412.

4. Cowan AQ, Cho DJ, Rosenson RS. Importance of blood rheology in the pathophysiology of atherothrombosis. Cardiovasc Drugs Ther. 2012;26(4):339-348.

5. Tzoulaki I, Murray GD, Lee AJ, Rumley A, Lowe GD, Fowkes FG. Relative value of inflammatory, hemostatic, and rheological factors for incident myocardial infarction and stroke: the Edinburgh Artery Study. Circulation. 2007;115(16):2119-2127.

6. Kikuchi Y, Sato K, Mizuguchi Y. Modified cell-flow microchannels in a single-crystal silicon substrate and flow behavior of blood cells. Microvasc Res. 1994;47(1):126139.

7. Kotani K, Satoh N, Yamada K, Taniguchi N, Shimatsu A. The influence of metabolic syndrome and chronic kidney disease on hemorheology assessed by the microchannel method. J Physiol Anthropol. 2010;29(5):157-160.

8. Hitsumoto T. Factors affecting impairment of blood rheology in obese subjects. J Cardiol. 2012;60(5):401-406.

9. Matsuo K, Ueda Y, Nishio M, Hirata A, Asai M, Nemoto $\mathrm{T}$, Kashiwase K, et al. Thrombogenic potential of whole blood is higher in patients with acute coronary syndrome than in patients with stable coronary diseases. Thromb Res. 2011;128(3):268-273.

10. Yoshida K, Kimura T, Aoki T, Tsunekawa K, Araki O, Shoho Y, Nara M, et al. Fasting serum insulin levels and insulin resistance are associated with blood rheology in Japanese young adults without diabetes. J Int Med Res. 2016;44(3):496-507.

11. Friedewald WT, Levy RI, Fredrickson DS. Estimation of the concentration of low-density lipoprotein cholesterol in plasma, without use of the preparative ultracentrifuge. Clin Chem. 1972;18(6):499-502.

12. Shirai K, Utino J, Otsuka K, Takata M. A novel blood pressure-independent arterial wall stiffness parameter; cardio-ankle vascular index (CAVI). J Atheroscler Thromb. 2006;13(2):101-107. 
13. Silberberg JS, Wlodarczyk J, Fryer J, Robertson R, Hensley MJ. Risk associated with various definitions of family history of coronary heart disease. The Newcastle Family History Study II. Am J Epidemiol. 1998;147(12):11331139.

14. Lloyd-Jones DM, Nam BH, D'Agostino RB, Sr., Levy D, Murabito JM, Wang TJ, Wilson PW, et al. Parental cardiovascular disease as a risk factor for cardiovascular disease in middle-aged adults: a prospective study of parents and offspring. JAMA. 2004;291(18):2204-2211.

15. Fujishima M, Kiyohara Y, Kato I, Ohmura T, Iwamoto H, Nakayama K, Ohmori S, et al. Diabetes and cardiovascular disease in a prospective population survey in Japan: The Hisayama Study. Diabetes. 1996;45(Suppl 3):S1416.

16. Hitsumoto T. Impact of hemorheology assessed by the microchannel method on pulsatility index of the common carotid artery in patients with type 2 diabetes mellitus. J Clin Med Res. 2017;9(7):579-585.

17. Hitsumoto T. Relationship Between Hemorheology Assessed Using Microchannel Array Flow Analyzer and Kidney Function in Hypertensive Patients. Cardiol Res. 2017;8(4):147-153.

18. Hitsumoto T. Association of hemorheology with highsensitivity cardiac troponin $\mathrm{T}$ levels in patients with type 2 diabetes mellitus assessed by microchannel array flow analyzer. Cardiol Res. 2017;8(6):304-311.

19. Lowery CL, 3rd, Elliott C, Cooper A, Hadden C, Sonon RN, Azadi P, Williams DK, et al. Cigarette Smoking-Associated Alterations in Serotonin/Adrenalin Signaling Pathways of Platelets. J Am Heart Assoc. 2017;6(5):e005465.

20. Alkan FA, Cakmak G, Karis D, Saglam ZA, Saler T, Temiz LU, Yenigun M, et al. The evaluation of plasma viscosity and endothelial dysfunction in smoking individuals. Clin Hemorheol Microcirc. 2014;58(3):403-413.

21. Shimada S, Hasegawa K, Wada H, Terashima S, SatohAsahara N, Yamakage H, Kitaoka S, et al. High blood viscosity is closely associated with cigarette smoking and markedly reduced by smoking cessation. Circ J. 2011;75(1):185-189.

22. Chei CL, Yamagishi K, Kitamura A, Kiyama M, Imano H, Ohira T, Cui R, et al. C-reactive protein levels and risk of stroke and its subtype in Japanese: The Circulatory Risk in Communities Study (CIRCS). Atherosclerosis. 2011;217(1):187-193.

23. Iso H, Noda H, Ikeda A, Yamagishi K, Inoue M, Iwasaki $\mathrm{M}$, Tsugane $\mathrm{S}$. The impact of $\mathrm{C}$-reactive protein on risk of stroke, stroke subtypes, and ischemic heart disease in middle-aged Japanese: the Japan public health centerbased study. J Atheroscler Thromb. 2012;19(8):756-766.

24. Ross R. Atherosclerosis - an inflammatory disease. N Engl J Med. 1999;340(2):115-126.

25. Libby P, Ridker PM, Maseri A. Inflammation and atherosclerosis. Circulation. 2002;105(9):1135-1143.

26. Soeki T, Niki T, Kusunose K, Bando S, Hirata Y, Tomita $\mathrm{N}$, Yamaguchi $\mathrm{K}$, et al. Elevated concentrations of pentraxin 3 are associated with coronary plaque vulnerability. J Cardiol. 2011;58(2):151-157.

27. Tzoulaki I, Murray GD, Lee AJ, Rumley A, Lowe GD,
Fowkes FG. Inflammatory, haemostatic, and rheological markers for incident peripheral arterial disease: Edinburgh Artery Study. Eur Heart J. 2007;28(3):354-362.

28. Podrez EA, Byzova TV, Febbraio M, Salomon RG, Ma Y, Valiyaveettil M, Poliakov E, et al. Platelet CD36 links hyperlipidemia, oxidant stress and a prothrombotic phenotype. Nat Med. 2007;13(9):1086-1095.

29. Qadir F, Alam SM, Siddiqi AQ, Kamran A. Pitavastatin is a potent anti-inflammatory agent in the rat paw model of acute inflammation. Pak J Pharm Sci. 2014;27(6 Spec No.):2169-2175.

30. Gounder VK, Arumugam S, Arozal W, Thandavarayan RA, Pitchaimani V, Harima M, Suzuki K, et al. Olmesartan protects against oxidative stress possibly through the Nrf2 signaling pathway and inhibits inflammation in daunorubicin-induced nephrotoxicity in rats. Int Immunopharmacol. 2014;18(2):282-289.

31. Janic M, Lunder M, Prezelj M, Sabovic M. A combination of low-dose fluvastatin and valsartan decreases inflammation and oxidative stress in apparently healthy middleaged males. J Cardiopulm Rehabil Prev. 2014;34(3):208212.

32. Lowe RN, Vande Griend JP, Saseen JJ. Statins for the primary prevention of cardiovascular disease in the elderly. Consult Pharm. 2015;30(1):20-30.

33. Foulquier S, Bohm M, Schmieder R, Sleight P, Teo K, Yusuf S, Schumacher H, et al. Impact of telmisartan on cardiovascular outcome in hypertensive patients at high risk: a Telmisartan Randomised AssessmeNt Study in ACE iNtolerant subjects with cardiovascular Disease subanalysis. J Hypertens. 2014;32(6):1334-1341.

34. Okura T, Watanabe S, Kurata M, Manabe S, Koresawa M, Irita J, Enomoto D, et al. Relationship between cardioankle vascular index (CAVI) and carotid atherosclerosis in patients with essential hypertension. Hypertens Res. 2007;30(4):335-340.

35. Masugata H, Senda S, Inukai M, Murao K, Himoto T, Hosomi N, Murakami K, et al. Association of cardio-ankle vascular index with brain natriuretic peptide levels in hypertension. J Atheroscler Thromb. 2012;19(3):255-262.

36. Hitsumoto T. Clinical Significance of Cardio-Ankle Vascular Index as a Cardiovascular Risk Factor in Elderly Patients With Type 2 Diabetes Mellitus. J Clin Med Res. 2018;10(4):330-336.

37. Satoh N, Kotani K, Wada H, Himeno A, Shimada S, Sasaki Y, Yamada K, et al. Unfavorable blood rheology is closely associated with arterial stiffness in obese patients. Endocr J. 2009;56(7):915-918.

38. Hitsumoto T. Relationship between cardiovascular risk factors and hemorheology assessed by microchannel method in patients with type 2 diabetes mellitus. Diabetology International. 2017;8(3):316-322.

39. Yagi H, Sumino H, Aoki T, Tsunekawa K, Araki O, Kimura T, Nara M, et al. Impaired blood rheology is associated with endothelial dysfunction in patients with coronary risk factors. Clin Hemorheol Microcirc. 2016;62(2):139150.

40. Endo K, Saiki A, Ohira M, Miyashita Y, Shirai K. Cardioankle vascular index may reflect endothelial function in 
type 2 diabetes. Int J Clin Pract. 2011;65(11):1200-1201.

41. Noike H, Nakamura K, Sugiyama Y, Iizuka T, Shimizu $\mathrm{K}$, Takahashi M, Hirano K, et al. Changes in cardio-ankle vascular index in smoking cessation. J Atheroscler Thromb. 2010;17(5):517-525.

42. Iwasa T, Amiya E, Ando J, Watanabe M, Murasawa T, Komuro I. Different contributions of physical activity on arterial stiffness between diabetics and non-diabetics. PLoS One. 2016;11(8):e0160632.

43. Yamada K, Hirayama T, Hasegawa Y. Antiplatelet effect of losartan and telmisartan in patients with ischemic stroke. J Stroke Cerebrovasc Dis. 2007;16(5):225-231.

44. van der Loo B, Spring S, Koppensteiner R. High-dose atorvastatin treatment in patients with peripheral arterial disease: effects on platelet aggregation, blood rheology and plasma homocysteine. Clin Hemorheol Microcirc. 2011;47(4):241-251.

45. Miyashita Y, Endo K, Saiki A, Ban N, Yamaguchi T, Kawana H, Nagayama D, et al. Effects of pitavastatin, a 3-hydroxy-3-methylglutaryl coenzyme a reductase inhibitor, on cardio-ankle vascular index in type 2 diabetic patients. J Atheroscler Thromb. 2009;16(5):539-545.

46. Miyoshi T, Doi M, Hirohata S, Kamikawa S, Usui S, Ogawa H, Sakane K, et al. Olmesartan reduces arterial stiffness and serum adipocyte fatty acid-binding protein in hypertensive patients. Heart Vessels. 2011;26(4):408-413. 